\title{
Clinical pathophysiology of thyroid eye disease: The Cone Model
}

\author{
Paul Meyer ${ }^{1,2} \cdot{\text { Tilak Das } \mathbb{D}^{3} \cdot \text { Nima Ghadiri } \mathbb{D}^{4} \cdot \text { Rachna Murthy }}^{5,6} \cdot$ Sofia Theodoropoulou ${ }^{7}$
}

Received: 21 November 2018 / Accepted: 22 November 2018 / Published online: 18 January 2019

(c) The Royal College of Ophthalmologists 2019

\begin{abstract}
The clinical features of thyroid eye disease are dictated by the orbit's compartmentalisation; particularly, the muscle cone, which is delimited by the rectus muscles, their inter-muscular septa and the posterior sclera. The cone is anchored to the orbit apex and contains the posterior globe, the muscle bellies, a fat pad, and the blood circulation, optic nerve, and CSF sheath. It is surrounded by mobile extraconal fat, retained by the orbital septum.

Thyroid eye disease is caused by expansion of muscle bellies and fat within the cone. Mechanical properties of the cone determine that the disease partitions into three phases: circumferential expansion, with forward displacement of extraconal fat; axial elongation, with increasing cone pressure; impedance of posterior venous outflow, with cone oedema and venous flow reversal.

Venous flow reversal can be observed in the conjunctival circulation. It is initially transient, accompanying rises in cone pressure caused by eye movements, but later becomes permanent. It is a useful clinical sign that locates diseased muscles and anticipates venous compressive crises.

Strabismus arises when inflamed rectus muscles, swollen by hydrated glycosaminoglycans, lose contractility and compliance. The incomitance is moderated by increasing stiffness affecting all the rectus muscles, as they are stretched during cone expansion.

Immunomodulation, which rapidly reduces cone volume, relieving muscle elongation and stiffness, may paradoxically unmask strabismus. However, ciclosporin A suppresses late post-inflammatory fibrosis and only 4 of 71 patients so-treated required strabismus surgery.
\end{abstract}

The cone model also accounts for the variety of clinical presentations of thyroid eye disease.

\section{Introduction}

The eye globe is balanced on a bed of fat and rotated by a conical array of rectus muscles. These contain the fat and are anchored to the orbital apex.

During thyroid eye disease, the rectus muscles become swollen by hygroscopic glycosaminoglycans (hyaluronic acid) and the fat also expands-all within a rigid orbit [1]. The relationship between these pathological changes and the clinical signs and symptoms of the disease has hitherto lacked clarity.

However, careful correlations between magnetic resonance imaging (MRI) scans and clinical findings reveal a sequence

Supplementary information The online version of this article (https:// doi.org/10.1038/s41433-018-0302-1) contains supplementary material, which is available to authorized users.

Paul Meyer

parm2@cam.ac.uk

Extended author information available on the last page of the article of mechanical events which predict the various presentations and the progression of thyroid eye disease. They are summarised by a simple model ("The Cone Model"), founded on the concept of the muscle cone as a discrete and expanding unit within a rigid orbit.

\section{Data sources}

Between 1992 and June 2017, there is a complete record of all clinical data from the 4453 patients who attended the Addenbrooke's Hospital Medical Ophthalmology clinic, and it resides on a fully searchable database. 528 of these patients were treated for thyroid eye disease.

The early patients in this series were imaged radiologically by computerised tomography (CT) scanning, but MRI scanning was introduced after 1996. In 2001, MRI became our standard imaging modality and we established a scanning protocol, which included coronal T1, T2, and STIR sequences. This paper is based on the 
results from 412 patients with thyroid eye disease, who were scanned on one or more occasions, according to the 2001 protocol.

From 2001, the direction of conjunctival venous flow in all quadrants was also recorded for both eyes on every clinic visit.

During this period, the management of all our patients has been consistent. In every case, suspected drives have been identified and withdrawn or treated. These included smoking cessation, restoration of endocrine control, and treatment of infections (particularly, Staphylococcus aureus colonisation of the upper respiratory tract-paper pending) and malignancy.

Those 177 patients whose thyroid eye disease had caused optic nerve compromise or severe motility disturbance were immunomodulated using intravenous methylprednisolone, tapering oral prednisolone, and calcineurin inhibition (mostly with ciclosporin A). This regime was devised in order to achieve immunomodulation with immediate effect, and to minimise late rectus muscle fibrosis.

Our first results were published in 2007 [2] and the expanded cohort has now been analysed, with quantification of fields of binocular single vision (BSV) (paper in preparation). The motility results, which support the cone model, are particularly remarkable and are discussed below.

\section{Physical principles}

\section{Solids and liquids}

The motion of molecules in a solid is restricted by attractive forces: energy is required to separate or displace them.
The molecules in a liquid (or gas) have random motion: energy is required to contain them.

\section{Pressure}

When liquid (or gas) is introduced into a container, its walls become stretched (extended) until the force extending them equals that required to constrain the contents. This force (per unit area) represents the pressure of the contents (Fig. 1). Every pressure differential requires deflection of a solid interface.

\section{Stress - strain curves}

For most structures, elongation (strain) in response to a stretching force (stress) is roughly linear; however, they fracture when loading is excessive [3]. In soft biological tissues, including rectus muscles (Fig. 2) [4], the linear stress-strain response is usually preceded by a phase in which minimal tension causes great elongation. This gives rise to a sigmoid stress-strain curve.

\section{Stress in the wall of a pressurised container depends on its geometry}

In a pressurised cylindrical structure, the circumferential wall stress is twice the axial stress. This means that, when the stress-strain curve of the wall is equal in all directions, the vessel will expand circumferentially, rather than elongate (Fig. 1): [3].

The muscle cone is roughly cylindrical and is further weakened circumferentially by longitudinal gaps between the muscles, bridged only by connective tissue septa. Therefore, during a rise in cone volume, circumferential expansion is strongly favoured over elongation.
Fig. 1 The distribution of stress in the wall of a pressurised vessel depends on its shape. If it is spherical, wall stress is uniform; when it is cylindrical, circumferential stress (S2) is twice the axial stress (S1) [3]

\section{Pressure and wall stress}
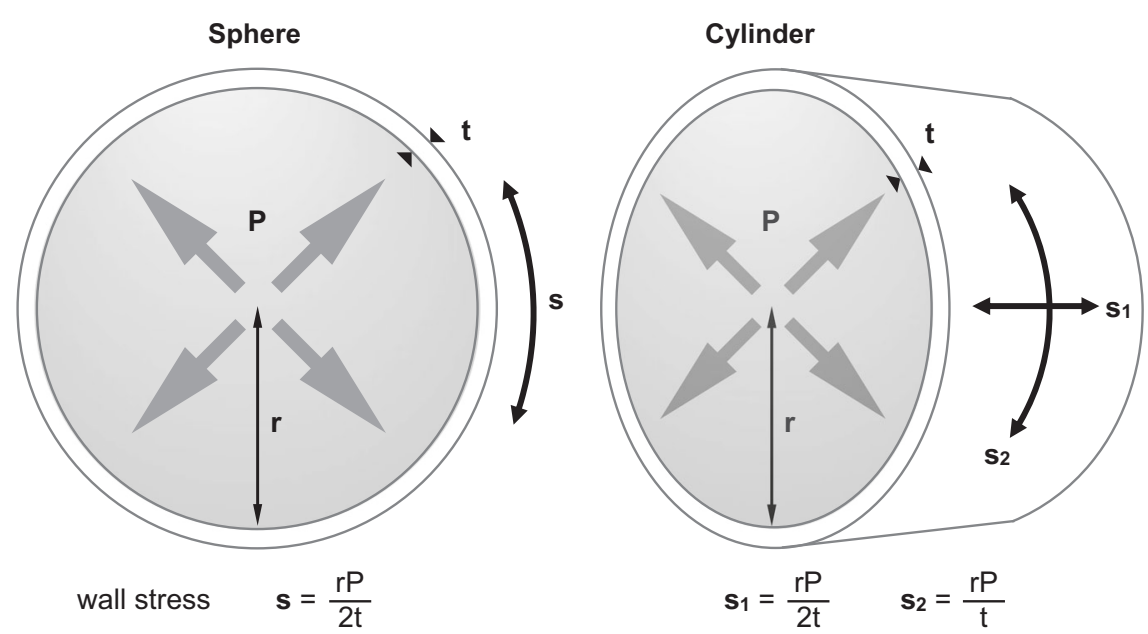


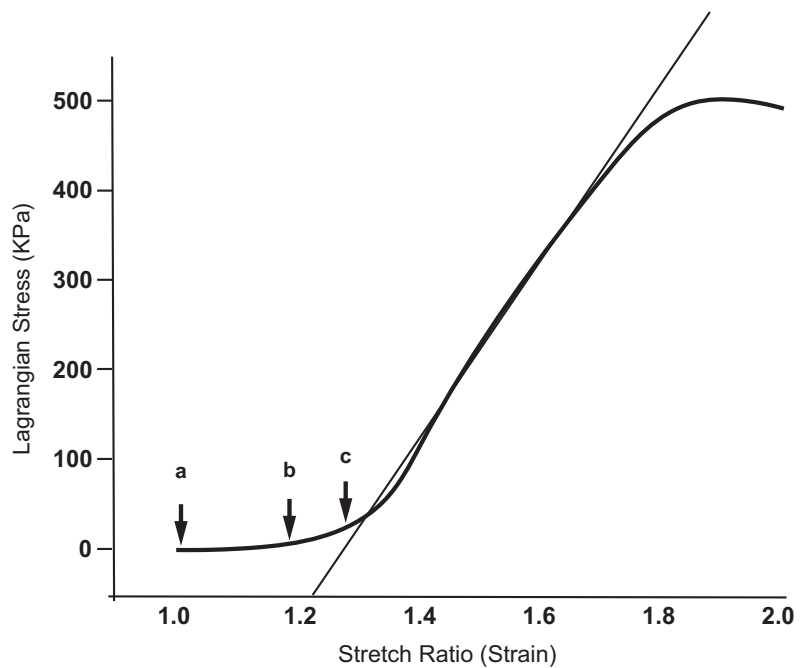

Fig. 2 Stress-strain function for normal bovine rectus muscle ex vivo (redrawn from Yoo et al. [4]). Increasing cone volume is accommodated by elongation of the rectus muscles $(a, b)$. As the linear phase of the stress-strain curve is approached, the rectus muscles are stretched until the force they return generates cone pressure approaching that in the superior ophthalmic vein (c)

\section{Elastic compartments of the normal orbit}

\section{The normal muscle cone}

The rectus muscles anchor the eye globe to the orbital apex (Fig. 3). They insert anterior to the equator of the globe; therefore, their sheaths and inter-muscular septa define a muscle cone, which is capped and indented by the posterior globe. As well as containing the muscle bellies and the larger part of the globe, this cone encapsulates the fat pad, which acts as a fulcrum, allowing the contraction of any rectus muscle to stretch its antagonists and rotate the eye. Normal eye movements demand that the cone is very compliant, changing shape continuously to rebalance tension within its walls.

The muscle cone and its contents are segregated from the remaining orbit by their connective tissue envelope. Outside this, it is surrounded by a further cushion of (extraconal) fat.

The cone also transmits various structures: particularly, the optic nerve, which is surrounded by a meningeal cuff containing cerebrospinal fluid (CSF), and the ophthalmic blood circulation. The ophthalmic artery, which delivers blood from the internal carotid artery, emerges from the optic foramen to enter the cone alongside the optic nerve.

Two ophthalmic veins drain blood posteriorly, into the cavernous sinus: the superior ophthalmic vein traverses the cone, but the inferior ophthalmic vein remains extraconal.

\section{Competition between compartments for space in a rigid orbit}

Most orbital diseases are accompanied by changes in the volume of one or more compartments within the orbit. But the orbit is rigid; therefore, there follows a jostling for space: as one compartment enlarges, the others accommodate it by displacement or volume reduction. In practice, closed or solid elements are displaced; compartments that communicate with extraorbital reservoirs can be emptied (for example, the extrusion of blood from veins or CSF from the optic nerve sheath).

Rearrangements of the orbit contents are mediated by the deflection of tissue interfaces.

Clinical pathology is determined by the sequence and magnitude of these events and the functional changes they cause.

\section{A hierarchy of pressures}

Redistribution of orbital fat occurs at very low pressure differentials [5]. Tiny displacements of extraconal fat take place to accommodate the momentary changes in cone pressure and shape that accompany eye movements. And this mechanism is the first to be recruited to accommodate pathological expansion of the muscle cone.

The fluid compartments in the normal orbit display a hierarchy of pressures, some of which can be measured; others, which can only be assumed.

The pressures in CSF $(7-15 \mathrm{mmHg})$, veins (intermediate between CSF and aqueous), aqueous (10-20 $\mathrm{mmHg}$ ) and arteries $(100-150 \mathrm{mmHg}$ ) predict a sequence in which open compartments would collapse as tension rises higher within the muscle cone.

\section{Retropulsion: a model for the displacement of extraconal fat}

Changes in cone pressure can be modelled, experimentally, by MR Imaging of fat redistribution during retropulsion of the globe. This alters the shape of the muscle cone, shortening it and increasing its circumference. As a result, extraconal fat is displaced forward (nasally, inferolaterally, and inferiorly), until the intermuscular septa meet the orbit wall. This displacement is opposed by increased tension within the orbital septum (see also [5]).

Once all fat surrounding the muscle cone has migrated forward at low pressure, further retropulsion of the globe would require expulsion of fluid from another pressurised compartment: CSF into the cranium, venous blood into the cavernous sinus, or aqueous into venous blood.

In clinical ophthalmology, we consider retropulsion to estimate the notional entity, "orbital tension". However, 

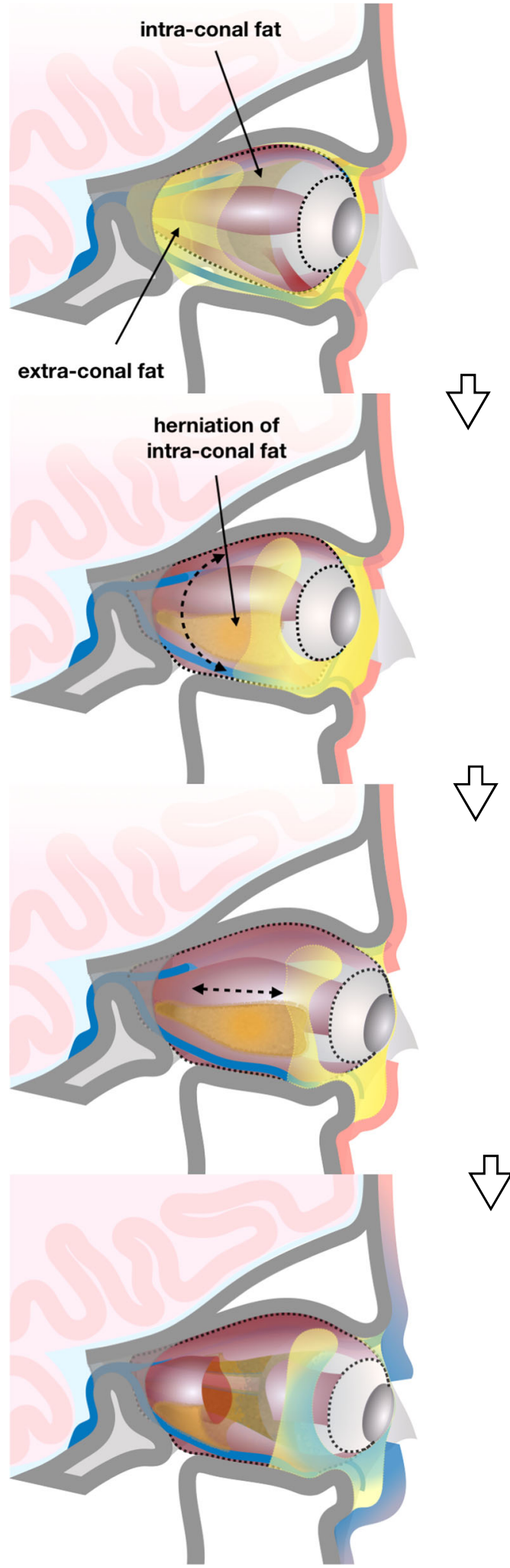

\section{Normal}

\section{Thyroid eye disease}

\section{Phase 1}

Expansion of rectus muscles and fat: cone enlarges circumferentially, until arrested by orbit walls. Extra-conal fat is displaced forward.

Phase 2

Axial advancement of globe: rectus muscles are stretched, therefore cone pressure rises.

\section{Phase 3}

(lateral rectus cut to illustrate oedematous cone fat and optic nerve stretch)

\section{High cone pressure impedes flow} through superior ophthalmic vein to cavernous sinus. Intra-conal fat oedema further elevates cone pressure. Conjunctival venous flow reversal. Oedema of extra-conal fat and eyelids.
Fig. 3 The cone model. Normal: the rectus muscles anchor the globe to the orbital apex. Connective tissue encapsulates the muscles and joins them (the intermuscular septa). This defines the muscle cone: ........... Venous drainage to the cavernous sinus passes both through the cone (superior ophthalmic vein) and outside it (inferior ophthalmic vein). The cone is surrounded by extraconal fat. Thyroid eye disease: $1 /$ circumferential enlargement of the muscle cone displaces extraconal fat; $2 /$ stretching of rectus muscles elevates cone pressure; 3/ high pressure impedes venous drainage to the cavernous sinus. 
the paragraphs above argue that there is no single orbital tension; rather, as many orbital tensions as there are compartments to displace or compress.

\section{The muscle cone in thyroid eye disease}

\section{Expansion and hypertension of the cone}

During thyroid eye disease, particular rectus muscles become swollen by hygroscopic glycosaminoglycans (Figs. 3 and 4) and fat also expands within the muscle cone. The cone can change shape, but remains a coherent anatomical unit: its enlargement requires the displacement or compression of other structures in the rigid orbit.

The clinical evolution of the disease resolves into three overlapping phases, reflecting the sequence of these changes as cone pressure rises.

Phase 1: circumferential expansion of the cone and displacement of extraconal fat. The wall of the muscle cone is weakened, circumferentially, by the intermuscular septa, and circumferential stress is greater than axial stress. Therefore, rising cone volume preferentially increases cone circumference until the rectus muscles and intermuscular septa meet the orbit walls (Figs. 3 and 5). Extraconal fat is displaced forward, stretching the orbital septum, which returns ever-increasing force, determining cone pressure at this stage.

Phase 2: axial advancement of the globe and stretching of rectus muscles. Extraconal fat is free to prolapse forward, but the cone contents are contained within a capsule, comprising the rectus muscles with their intermuscular septa and the posterior globe. Therefore, once the cone has occupied all available space in the posterior orbit, further increases in its volume must, inevitably, advance the globe, elongating the rectus muscles.

Their stress-strain curve ex vivo [4] (Fig. 2) shows that muscle elongation is initially associated with just a small rise in stress, returning a minimal increase in cone pressure. However, when the steep phase of the curve is approached, small increments in length (strain) generate enormous increases in stress, resulting in very high cone pressures.

Published stress-strain curves in vivo from innervated human rectus muscles affected by thyroid eye disease [6] indicate that muscle tone may be high, steepening the curve and reducing the elongation (hence proptosis) needed to elevate cone pressure.

Phase 3: impeded posterior venous drainage and reversal of conjunctival venous flow. A critical stage arises when cone pressure approaches venous pressure during eye movements and posterior venous drainage to the cavernous sinus is impeded. Arterial perfusion continues, and pressure within orbital microcirculations may be expected to rise
Fig. 4 Venous compressive crisis: coronal STIR MRI scan (cuts immediately behind the globes, then $8 \mathrm{~mm}$ apart). Note the high STIR signal from swollen, inflamed rectus muscles. The muscles with overlying conjunctival venous flow reversal are shown in the middle pane $(*)$

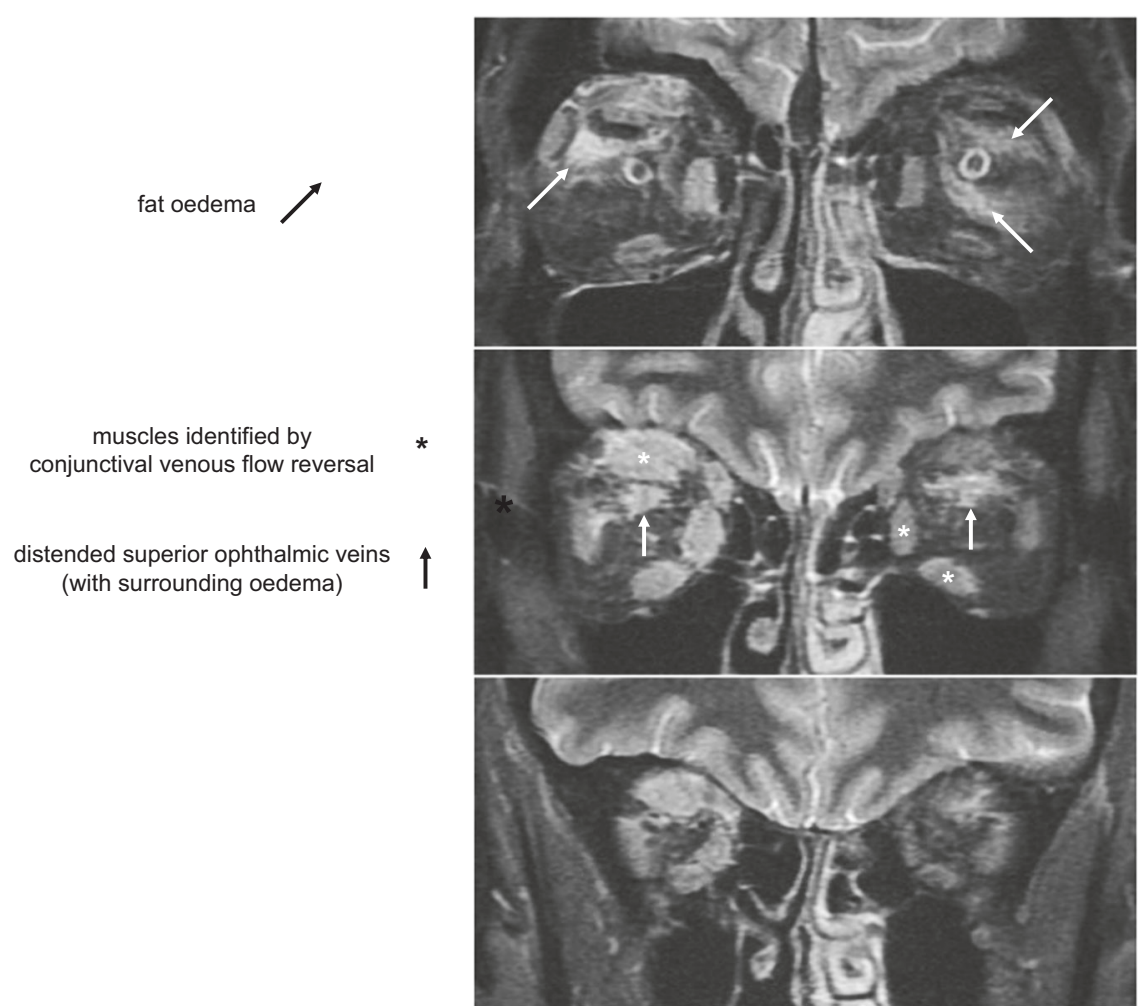




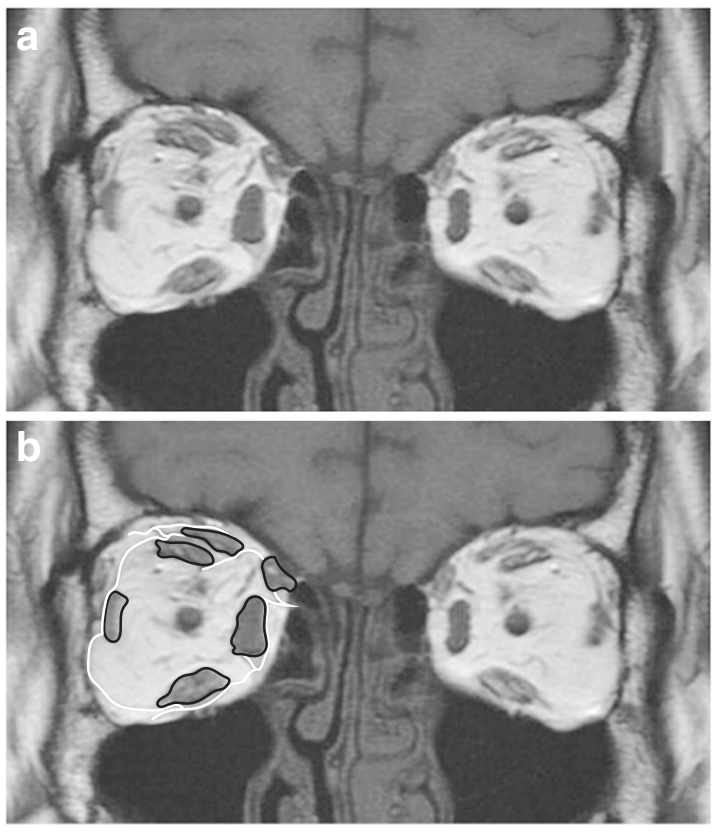

Fig. 5 a Unannotated. b Rectus muscles outlined (black); intermuscular septa outlined (white). Venous compressive crisis: coronal T1 MRI scan (same patient as Fig. 4: level 1 only). Note the inferotemporal herniation of cone fat: cone has expanded until its arrest by walls of the orbit

enormously. These leak plasma, with consequent oedema (Fig. 4), hence a further increase in cone volume and pressure: a venous compressive crisis.

Only the redirection of venous drainage can resolve this physiologically. This is possible because the orbital veins lie at a watershed between the cavernous sinus and facial circulation, and the direction of their drainage is determined according to the relative pressure gradients.

When high cone pressure impedes posterior orbital venous drainage, redirection of flow can be recorded, clinically, in the conjunctival circulations (Figs. 6; 7; video). Initially, it takes place only during eye movements, but as the disease progresses it becomes constant. On occasion, the development of stable conjunctival venous flow reversal may signify spontaneous decompression of a hypertensive orbit.

\section{Clinical corollaries of the cone model}

Once the expanded muscle cone has displaced all available extraconal fat, it is arrested by the bony orbit wall and the orbit feels firm to retropulsion.

Further expansion of the cone extends the rectus muscles at low stress until they reach the steep section of their stress-strain curves, after which stress (hence, cone pressure) rises sharply.
This cascade of events is expressed in the symptoms and signs of thyroid eye disease.

\section{Conjunctival venous flow reversal}

Anterior conjunctival venules are easy to identify: they are large $(10-50 \mu \mathrm{m})$, radial vessels, with non-pulsatile flow, containing aggregated erythrocytes, and striation of the erythrocyte column occurs where they converge. They normally drain anteriorly into the limbal venous circle, which delivers blood into radial episcleral collecting veins [7], en route to the superior and inferior ophthalmic veins and cavernous sinus. Communications between anterior and posterior conjunctival veins do exist, but they rarely flow under normal circumstances.

However, when pressure in the muscle cone approaches that in orbital veins, episcleral venous pressure also rises, causing conjunctival venous flow to reverse, as it is diverted into posterior tarsal veins and the eyelid circulation (see Figs. 6a, b; 7; video). This is initially transient, during eye movements: dynamic flow reversal (video). It is a first indication that the posterior drainage of orbital venous blood is becoming compromised by elevated cone pressure, and it heralds the risk of a venous compressive crisis.

This sign can also inform us about the distribution of pathology among the rectus muscles. An eye movement is initiated when contraction of a rectus muscle elevates pressure in the cone sufficiently to extend its antagonist. Muscles that are inflamed or infiltrated (with glycosaminoglycans or connective tissue) offer greater resistance to extension. Therefore, stretching of diseased antagonists will give the highest rise in cone pressure and the highest probability of flow reversal.

Clinical examination of conjunctival venous flow reversal is undertaken using green light. It aims to establish, for each rectus muscle in turn, whether its extension elevates cone pressure sufficiently to divert venous drainage.

The patient gazes away from the field under examination (Fig. 7); therefore, the muscle corresponding to that field is an antagonist under tension, and the observations reflect the resulting elevation of cone pressure. Conjunctival venous flow reversal (blood flowing away from the limbus) is most likely to arise in circulations overlying stiffer, diseased muscles (Fig. 4).

In a study of 66 coronal MRI scans from patients with thyroid eye disease (paper in preparation), we correlated the location of this sign with ranking of the intensity of the T2 signal from rectus muscles (indicating free water; hence, inflammation) in contemporaneous coronal STIR MRI scans. It arose within $90^{\circ}$ of the most actively inflamed muscle in $88 \%$ of eyes and identified that muscle in $61 \%$ of eyes. 
Fig. 6 a Primary position. b Forced gaze away from circulation under examination stretches the underlying rectus muscle. Conjunctival veins normally flow into the limbal venous circle and episcleral collecting veins. The more stretched the muscle cone, the less compliant the muscles become. Contraction of a rectus muscle can no longer extend its antagonist sufficiently and rotation of the globe is limited. However, cone pressure rises greatly: episcleral collecting veins become compressed and conjunctival venous flow reverses

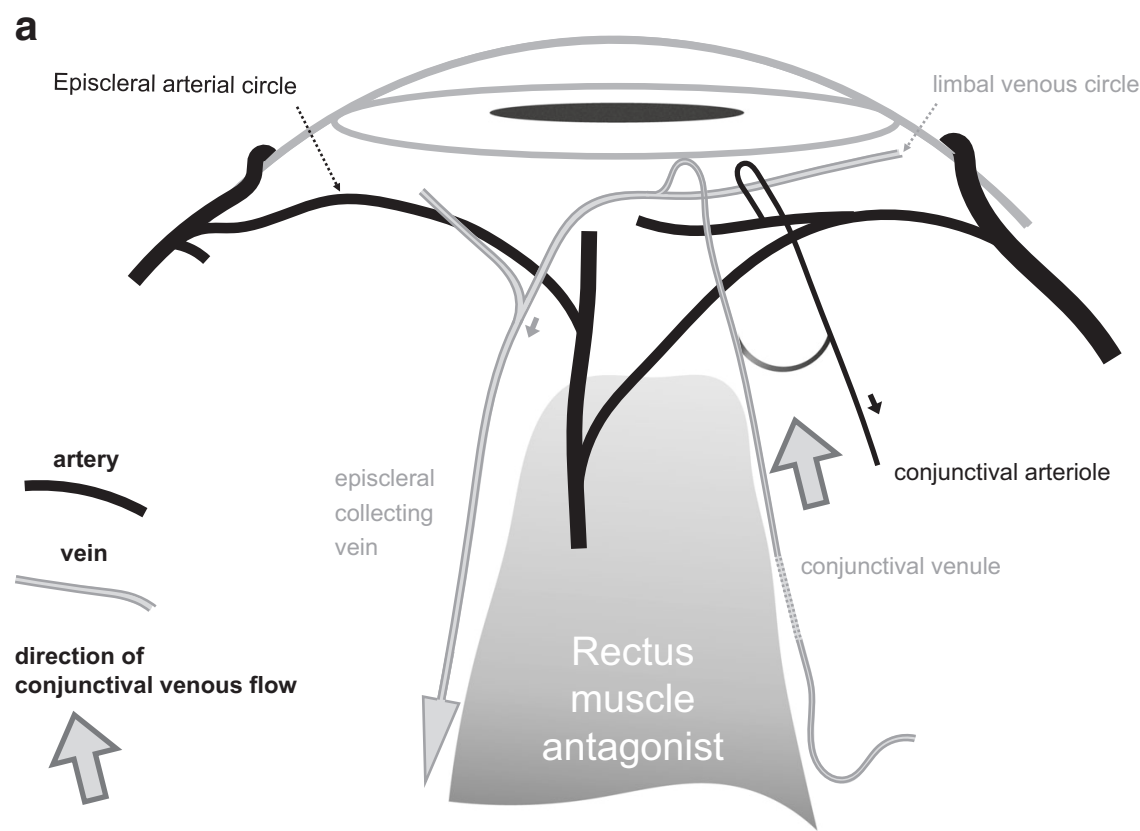

b

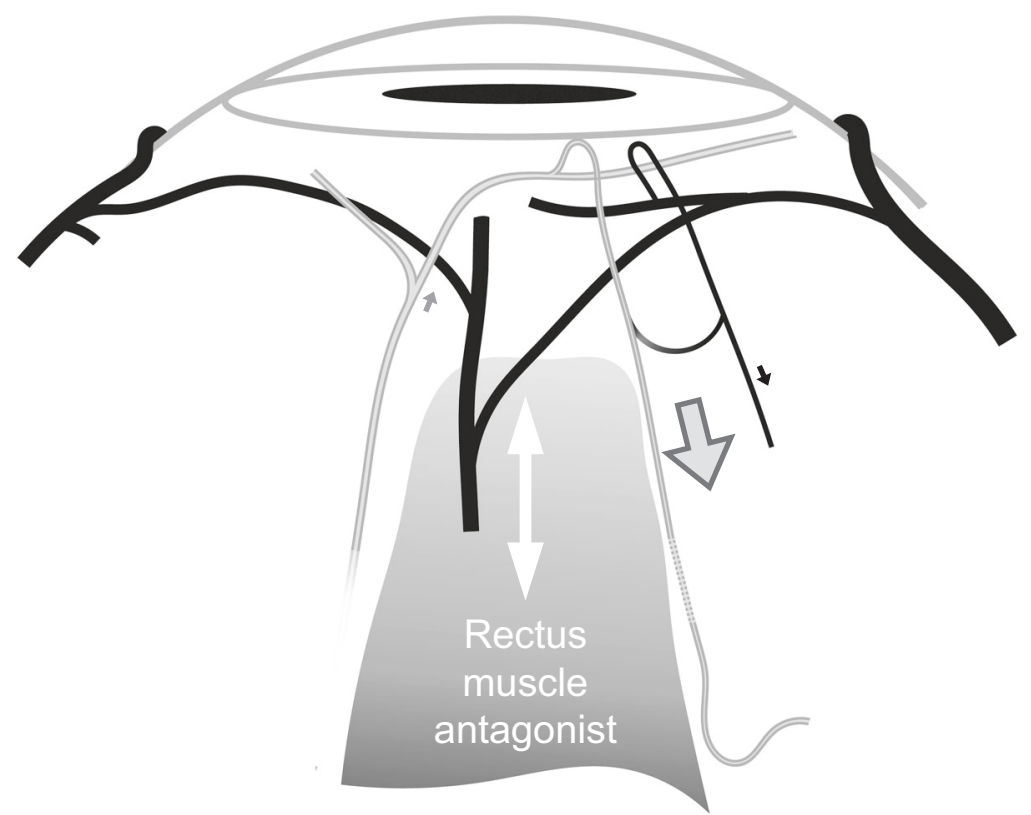

\section{Cone pressure during venous hypertensive crisis}

Conjunctival venous flow reversal indicates that orbital and episcleral veins lie at a watershed, drainage switching from the cavernous sinus to the facial veins at higher cone pressures. Pressure in orbital veins normally exceeds CSF pressure and, if complete closure of the superior ophthalmic vein were required to reverse venous drainage, the cone pressure should also empty CSF from the optic nerve sheath into the cranium.

However, examination of the scans from ten patients with orbital hypertensive crises identified just a single eye in which this had happened (Fig. 8). She was a woman with "benign intracranial hypertension" and a lumbar puncture, performed shortly after the scan, gave an intracranial pressure of $20 \mathrm{mmHg}$. Intraocular pressure (presumed to be close to cone pressure) was $32 \mathrm{mmHg}$.

Since expulsion of CSF from the optic nerve sheath is rare in hypertensive orbits, the threshold cone pressure for redirection of venous blood must lie below CSF pressure and still further below orbital venous pressure. This confirms that venous flow reversal represents disturbance of the pressure differential between the facial veins and cavernous sinus, not complete superior ophthalmic vein occlusion. 


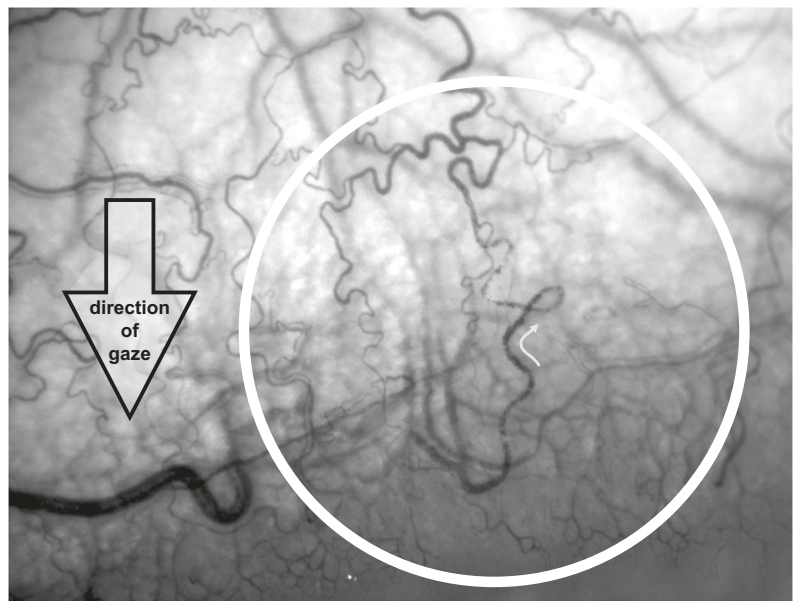

Fig. 7 Conjunctival venous flow reversal over a superior rectus muscle (please see video). White arrow illustrates reversal of the normal direction of venous flow, triggered by down-gaze

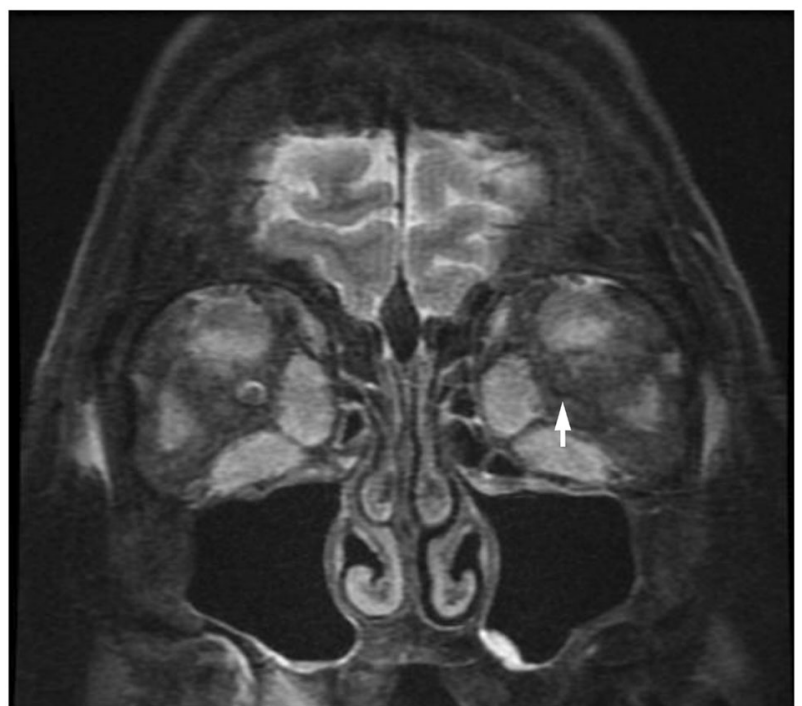

Fig. 8 Coronal STIR MRI during venous hypertensive crisis complicating thyroid eye disease, showing expulsion of CSF from optic nerve sheath (arrow). Patient had benign intracranial hypertension. Intraocular pressures: Right $30 /$ left $32 \mathrm{mmHg}$. Intracranial pressure: $20 \mathrm{mmHg}$

\section{Stiffness and disordered eye movements}

When a rectus muscle contracts, the extension of its antagonist is achieved by a momentary increase in cone pressure. Greater increases in pressure are needed to stretch inflamed or fibrosed muscles. Such muscles also generate less contractile force.

Expansion of the cone places all the rectus muscles under tension. Once they engage the steep section of their stressstrain curves, enormously higher forces are needed to stretch them: the cone becomes stiff. From then on, contraction of any rectus muscle further increases cone pressure, hence the stiffness of every muscle, and paradoxically inhibits eye movements.

Therefore, in thyroid eye disease, motility disturbances have three distinct mechanisms:

Inequality of stress-strain characteristics due to inflammation or fibrosis.

Loss of power in inflamed or fibrosed muscles.

Generalised stiffness, caused by elongation (increasing strain) of all rectus muscles, due to cone expansion and hypertension.

\section{Eye movements and the treatment of thyroid eye disease}

Intravenous steroids usually reduce cone volume with almost immediate effect, and stiffness of the muscle cone abates, with swift restoration of wide-angle deviations. This may deteriorate BSV, presumably by unmasking the underlying asymmetry of power and stress-strain responses, caused by inflammation and fibrosis.

Immunomodulation addresses chronic inflammation and, when calcineurin inhibitors are used, averts late fibrosis. This gradually reduces the asymmetry of muscle compliance, with late recovery of BSV.

These two phases of the response to immunosuppression are illustrated by our clinical cohort of 71 patients with severe motility disturbance, treated with intravenous methylprednisolone and oral ciclosporin A (paper in preparation). Quantification of fields of BSV (Fig. 9) showed many cases of deterioration immediately after starting treatment; however, the long-term (40 months) outcomes were excellent, with only four patients requiring strabismus surgery.

\section{The Cone Model and the clinical spectrum of thyroid eye disease}

Presentations of thyroid eye disease vary widely, but there is a recognisable clinical spectrum. At one extreme, patients suffer from severe proptosis, with a risk of optic nerve stretch and of ocular surface damage; at the other extreme, proptosis is modest and "orbital" (=cone) hypertension, with optic nerve dysfunction, occurs early.

These widely different constellations of symptoms confound clinical activity scores, but are accommodated by the overlapping phases of the cone model.

In Phase 1, forward displacement of extra-conal fat by an expanding cone is associated with periorbital swelling; however, there is little if any proptosis. The corresponding cone pressure is determined by tension in the 
field of BSV: 71 patients with severe motility disturbance

(Rx: iv Methylprednisolone, oral prednisolone, ciclosporin A)

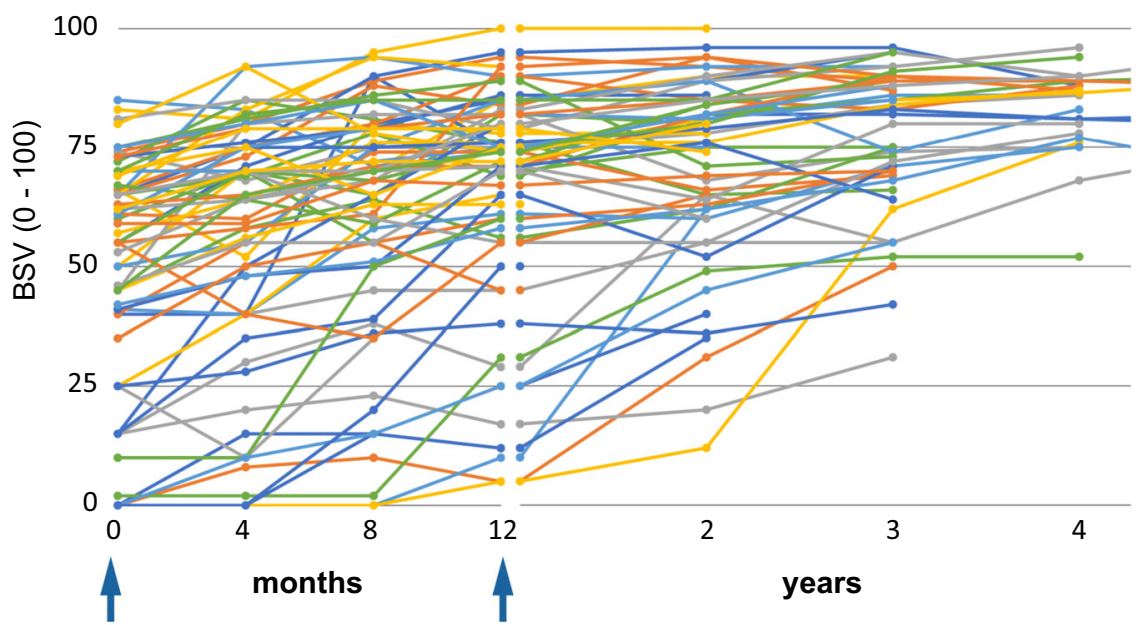

Fig. 9 Fields of BSV: 71 patients with severe motility disturbance (Rx: iv Methylprednisolone, oral prednisolone, and ciclosporin A). Incomitance arises in thyroid eye disease when particular muscles can no longer relax or contract in order to maintain BSV (due to inflammation, hydrated glycosaminoglycans, and increased muscle bulk). Stretching of the rectus muscles (which accompanies phase 2 of the disease) reduces muscle compliance globally and should, therefore, moderate such disturbances. Immunomodulation causes a rapid drop in cone pressure, negating the global component of motility restriction and lifting the suppression of myopathic strabismus. For the group as a whole, BSV began to improve immediately after immunomodulation; however, many patients deteriorated initially, as predicted. The antifibrotic effect of cyclosporin A did prevent scarring of damaged muscles during recovery, and strabismus surgery was required in only four patients

orbital septum. Asymmetrical myopathy may cause diplopia, due to loss of rectus muscle compliance and/or power.

Phase 2 begins when, having displaced extra-conal fat, the enlarging cone occupies all available space in the posterior orbit. Retropulsion at low pressure is no longer possible and the orbit feels firm.

The relationship between cone pressure and proptosis is entirely contingent on the stress-strain functions of the rectus muscles. Steeper curves can be expected when muscles are inflamed or fibrosed and these mechanical characteristics determine the amount of proptosis that can occur before phase 3 is entered.

Ultimately, the elevation of cone pressure during eye movements is sufficient to divert venous drainage from the cavernous sinus to the facial veins, demonstrable as conjunctival venous flow reversal. Stiffness of the cone begins to restrict eye movements, causing (but sometimes constraining) diplopia.

Phase 3 is the climax of this disease, and represents a mismatch between orbital arterial perfusion and venous drainage. Fat-suppressed T2 images of the orbit show oedema surrounding and emanating from the superior ophthalmic vein.

The diversion of orbital venous drainage into the eyelid microcirculations causes periorbital oedema. If shunting is sufficient, a new steady state may arise; however, cone pressure usually increases progressively, leading to episcleral venous hypertension, ocular hypertension and optic nerve dysfunction.

The posterior globe lies within the muscle cone; aqueous drains into episcleral venous blood and the superior ophthalmic veins, and these traverse the cone to reach the cavernous sinus. Although, intraocular pressure must always exceed cone pressure, they converge as cone pressure rises.

The clinical spectrum of thyroid eye disease is mainly determined by the evolving stress-strain functions of each patient's rectus muscles.

\section{Summary}

The muscle cone functions as a discrete anatomical entity, anchored to the apex of the rigid orbit. The clinical presentation of thyroid eye disease is a consequence of cone expansion, due to increasing bulk of the rectus muscles and fat that it contains.

Evolution of the disease partitions into three phases:

1. Circumferential cone expansion with displacement of extraconal fat.

2. Axial elongation of the cone, with muscle strain returning increasing stress, leading to:

3. Cone hypertension and stiffness, with impedance of venous drainage to the cavernous sinus. 
Conjunctival venous flow reversal is an objective clinical sign, which identifies the rectus muscles that are most affected by thyroid eye disease and signals transition to the high-risk hypertensive phase of the disease.

The cone model describes the evolution of thyroid eye disease in objective terms of structure and function. It allows symptoms and clinical or radiological signs to be used rationally to conceptualise orbital pathology, predict its evolution and guide its management.

\section{Compliance with ethical standards}

Conflict of interest The authors declare that they have no conflict of interest.

\section{References}

1. Khong JJ, McNab AA, Ebeling PR, et al. Pathogenesis of thyroid eye disease: review and update on molecular mechanisms. Br $\mathrm{J}$ Ophthalmol. 2016;100:142-50.

2. Meyer PAR. Avoiding surgery for thyroid eye disease. Eye. 2006;20:1171-7.

3. Gordon JE. Structures. Penguin: UK, 1978.

4. Yoo L, Kim H, Gupta V, Demer J. Quasilinear viscoelastic behavior of bovine extra ocular muscle tissue. Invest Ophthalmol Vis Sci. 2009;50:3721-8.

5. Frueh BR. Graves' eye disease: orbital compliance and other physical measurements. Trans Am Ophthalmol Soc. 1984;82: 492-598.

6. Simonsz HJ. Force-length recording of eye muscles during localanaesthesia surgery in 32 strabismus patients. Strabismus. 1994;2: 197-218.

7. Meyer PAR. Re-orchestration of blood flow by micro-circulations. Eye. 2018;32:222-9.

\section{Affiliations}

\section{Paul Meyer ${ }^{1,2} \cdot$ Tilak Das $\mathbb{1}^{3} \cdot$ Nima Ghadiri $\mathbb{1}^{4} \cdot$ Rachna Murthy ${ }^{5,6} \cdot$ Sofia Theodoropoulou ${ }^{7}$}

1 Consultant Medical Ophthalmologist (retired), Addenbrooke's Hospital, Cambridge, UK

2 Department of Engineering, Cambridge University, Cambridge, UK

3 Consultant Radiologist, Addenbrooke's Hospital, Cambridge, UK

4 Medical Ophthalmology trainee, Norfolk and Norwich Hospital, Norwich, UK
5 Consultant Oculoplastic Surgeon, Ipswich, UK

6 Thyroid Eye Disease Service, Addenbrooke's Hospital, Cambridge, UK

7 Trainee Ophthalmologist, Bristol Eye Hospital, Bristol, UK 